\title{
REVIEW
}

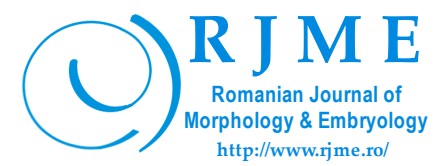

\section{Brain changes in depression}

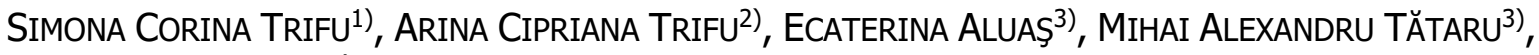 \\ RADU VIRGIL COSTEA ${ }^{4}$
}

\author{
1) Department of Neurosciences, Carol Davila University of Medicine and Pharmacy, Bucharest, Romania \\ 2) Department of General Medicine, Medical Military Institute, Bucharest, Romania \\ 3) Department of Psychology, University of Bucharest, Romania \\ 4) Department of General Surgery, Carol Davila University of Medicine and Pharmacy, Bucharest, Romania
}

\begin{abstract}
The present review addresses major depressive disorder (MDD) and the implications of antidepressant treatment in the field of brain neuroplasticity, an effect initially considered adjacent but currently passed as central in the process of remission of MDD. Both in experimental animal studies and in human studies in subjects with mood disorders, neuroplasticity is considered the fundamental mechanism of neural defense against stress. Stress is the mediator between neurofunctional, neuroendocrine, neurobiological and neuroimmune disorders and depressive pathology of various intensities. Neurons have a high potential to adapt to the influences of internal and external factors. We are talking about neuroplasticity at different levels: structural neuroplasticity involving adult neurogenesis (such as plastic changes, dendritic reconstruction, when the morphology of the spine is affected); synaptic functional neuroplasticity and molecular and cellular mechanisms involved. These two major dimensions explain the pathophysiology of depression, as well as the convergence of the mechanisms involved in stress, major depressive decompensations, and the concept of neuroplasticity as the present target for new effective and potent antidepressant treatments.
\end{abstract}

Keywords: antidepressants, neuroplasticity, prefrontal cortex, hippocampus, cognition.

\section{Introduction}

Hypostases and intensities of the depressive phenomenon:

- Major depressive disorder (MDD) is known to be defined by at least two weeks in which the underlying thymic mood is fallen and represents a significant change, noticed by the person in question or his entourage, compared to the patient's natural mood. In parallel, MDD involves decreased self-esteem, loss of interest and pleasure in previously pleasurable activities, reduced vitality, motivational-volitional unevenness, and pain without a clear cause (the concept of hidden depression) [1]. Patients with MDD may occasionally develop false beliefs, such as irrational beliefs, or they may overlap perceptual phenomena, such as accusatory and hallucinatory auditory hallucinations. There are patients with major depressive episodes spaced by significantly long intervals, in which the person in question works eutymically (recurrent depressive disorder), as well as clinical situations in which those in question may have symptoms for 2-3 years (more common chronic depression in men and over the age of 60). MDD negatively affects quality of life, outstanding job potential, educational, professional, or academic success, sleep and eating behaviors and health [2]. A percentage of $2-8 \%$ of adults that are diagnosticated with MDD go to suicide and die, and a percentage of $50 \%$ of adults who commit suicide have had MDD or have been diagnosticated with a mood disorder. Adjacent symptoms include delusions, concentration disorders, stability and selectivity of attention, fixation hypomnesia, withdrawal from social situations, decreased libido, irritability, recurrent and intrusive thoughts of death [3, 4]. Awakening insomnia is a typical symptom of a major depressive episode and is accompanied by marked anxiety, especially when the pattern of the episode is with melancholy features or psychotic phenomena.

- Psychotic depression, classically called depressive psychosis, involves a major depressive episode that associates delusional ideation, mostly guilt and guilt and/or visual illusions or auditory hallucinations (voices of deceased loved ones shouting at the person in question). Psychotic depression may occur in a recurrent depressive disorder or in a bipolar affective disorder $[5,6]$.

- Melancholic depression has noradrenergic coloration being characterized by athymhormia, loss of pleasure for most activities, failure to react to previously pleasant stimuli, a mood altered in the sense of sadness significantly more pronounced than moral pain or loss. The characteristics are severe intensity of symptoms in the first part of the day, awakening insomnia, bradykinesia, significant weight loss with absence of hunger (differential diagnosis with anorexia nervosa in which hunger is present) and feelings of guilt developed and felt in excess [7].

- Atypical depression has as fundamental axis paradoxical anhedonia and a pseudo-positivity. This refers to a significant weight gain in parallel with the increase in appetite and the feeling of mental comfort and peace with each meal. We also encounter hypersomnia as a defense mechanism, subjective sensation of heaviness in the limbs known as lead paralysis, feelings of social depreciation

This is an open-access article distributed under the terms of a Creative Commons Attribution-NonCommercial-ShareAlike 4.0 International Public License, which permits unrestricted use, adaptation, distribution and reproduction in any medium, non-commercially, provided the new creations are licensed under identical terms as the original work and the original work is properly cited. 
inconsistent with reality and hypersensitivity to the alleged interpersonal rejection that they perceive [8].

- Catatonic depression is a form of MDD, that is severe and involves significant alteration of motor behavior with mutism, akinesia, stupors change of consciousness, immobility, or the presence of aimless movements, sometimes bizarre. The differential diagnosis is made with catatonic schizophrenia, manic episodes that can be suddenly complicated by stupor or malignant neuroleptic syndrome.

- Dysthymia, also called persistent depressive disorder (PDD), in which the same symptoms appear in the physical area, as well as problems in the cognitive sphere as in depression, but these are of a lower intensity and longer duration [9]. During the period of two years of mood swings, we find a maximum of two months, the difference between dysthymia and major depressive episode being that in the first case two out of six symptoms are sufficient for diagnosis, while in MDD are necessary four of nine criteria, of which mandatory are anhedonia, concerns for uselessness, guilt or suicide, the presence of motor pathology that can vary between slowness and agitation [10].

- Recurrent brief depression (RBD) is defined by intermittent depressive episodes unrelated to menstrual cycles (in women). During a year, there may be between 6-12 such episodes, each meeting the criteria for MDD except for duration (in MDD the change in mood in the depressive sense is for at least 14 days, while for RBD, it is between 5-7 days).

- Minor depressive disorder has more than two symptoms characteristic of depression at the same time, being also a mood disorder.

- Adaptation disorder occurs when the person in question has significant difficulty adapting to cope with a significant psychosocial stressor. The subject reacts through inadequate responses that involve seemingly natural emotional and behavioral reactions, but which are more intense than the broad boundaries of normalcy, causing marked psychological distress, recurring concerns about the stress that caused the adjustment disorder, quasicontinuous recollections and negative inferences about the consequences, facts that ultimately affect the functionality.

- Premenstrual dysphoric disorder (PMDD) affects between $1.8-5.8 \%$ of women with active sexual life and is linked to the premenstrual syndrome, being a more severe and disabling form [11]. PMDD if formed of a sum of affective, somatic, and behavioral symptoms that reappear in each the moon, corresponding to the luteal phase of the menstrual cycle. Women characterized by PMDD will have the symptoms described above from adolescence to menopause, except in situations where they enter into hypothalamic amenorrhea or during pregnancy or lactation.

- Depression with peripartum onset is an intense depression that leads to disability, sometimes felt only after birth, but often, at a close look, symptoms are identified during pregnancy. In Diagnostic and Statistical Manual of Mental Disorders, fourth edition, text revision (DSMIV-TR), it was classified as postpartum depression, the terminology being changed to DSM-V in order not to exclude the cases of women who were depressed even during pregnancy [12]. We are talking about unspecified depressive disorder with peripartum onset, because it turned out that in over $50 \%$ of cases, the clinically evident postpartum onset had actually taken place before birth. The increase in the number of women who want to carry a pregnancy with the risk of depressive decompensation determines etiological and pathophysiological searches that allow extensive understandings for the complex approach to the depressive phenomenon, being known the association of depression with various neurobiological changes [13].

\section{Cerebral neuroplasticity}

The brain has a high level of plasticity if approached in a certain moment of time. The care environment and the prominent attachment figures from childhood, substantially influence the development of the brain. Preclinical studies were performed in rats that involved a pattern of maternal deprivation. These studies found that there is an association between impaired hypothalamic-pituitary-adrenal (HPA) function and changes of the density of glucocorticoid receptors (GRs). In parallel, there was a decrease in dendritic branches and a decrease in density in the spine, as well as a deterioration of synaptic transmission with potentiation and signal delay in the hippocampus. Childhood opposition disorder is a well-known risk factor for the person in question to develop depression in adulthood because, generally, is based on loss, neglect, or abuse [14].

Over the past decade, research has focused on the interaction between genetic and environmental factors in the clinical expression of depression. The interaction between heredity and the environment determines a longterm programming of gene expression, a possibility to change the expression of gene function even if the sequence itself remains unchanged. The phenomenon is known as epigenetic change, the process in question mediating the effects of environmental and social environment in childhood on gene expression. An example would be deoxyribonucleic acid (DNA) methylation that can persist into adulthood and can dictate personal vulnerability to the psychopathological area by influencing the activity of the HPA axis. The activity of above-described axis consists in regulating the stress response [15]. Regarding the neurobiology of depression, there are clearly different areas of the brain in which we find hyperactivity: amygdala, striated nucleus, limbic and subcortical regions. The recent conceptualization of depression is done in terms of a network dysfunction in terms of corticolimbic loops. The abovedescribed hyperactivity normalizes after antidepressant treatment and corresponds to the clinical improvement of symptoms. Like medication, psychotherapy alters brain function, although the neural correlations developed in depressive psychotherapy are less studied. In psychotherapy, initially, hyperactivity was found in the limbic and subcortical regions, later, with the increase of the number of sessions, in the same regions reporting hypoactivity [16].

Neural neuroplasticity involves the ability of the nervous system to modify its activity based on an intrinsic and extrinsic stimulus, to reorganize itself from structural, functional point of view and its connections. A fundamental property of neurons is their ability to alter their power in terms of the efficiency of synaptic transmission through a diverse number of mechanisms. This process depends on the actions and activities performed by the subject and is called synaptic plasticity. In the last century, it was 
considered to be a characteristic of different species of insects, currently being noted as a fundamental property of the human nervous system. The psychological modalities of cognitive training specific to different superior gnostic functions address synaptic plasticity as an intrinsic function of brain development and target the learning and memory processes. Redoubtable specialists are those who correctly appreciate the time windows in which plasticity can be maximally optimized by reshaping neural interconnectivity. Synaptic changes during learning and memorization lead to cognitive leaps in development and can be the premise of effective protocols for quality academic learning. Studies in human tissue indicate that synaptic plasticity could be a mechanism involved in the process of learning and memory, even though the real mechanism is still insufficiently studied.

\section{ㅁ Neurobiology of depression}

Severe depression is a serious illness with a significant risk of suicide. The World Health Organization (WHO) estimates that by 2020 depression will be number two on the Global Burden of Disease list [17]. MDD is debilitating through biological, cognitive, and psychological symptoms (we are talking about a double depressionpsychological reaction of depressive coloration superimposed on a major endogenous depressive episode) with personal and social consequences, with high levels of morbidity and mortality [18]. Depression affects between $10-15 \%$ of the general population throughout life, the biological bases being complex, with interaction disturbances that affect both neurons and glial cells in certain areas of the brain. Depressive symptoms include anhedonia, lack of self-worth, anergy, poor concentration, changes in appetite, along with motor changes, recurrent thoughts of death, all based on dysfunction of the neural network. At the molecular level, several biological processes are involved in depression, without limitation to changes in monoaminergic neurotransmitters to changes in the hormonal homeostasis of stress. Other mechanisms involved are reduced neurotrophic support, metabolic dysfunction, impaired immune response with increasing inflammation, oxidative stress, and mitochondrial dysfunction. Depression affects the plasticity of the brain and synaptic functions. Similar changes were found during a major depressive episode with those during aging, which led to the hypothesis that major depression could be associated with accelerated aging [19]. A meta-analysis performed for patients with clinical depression took into account as criteria for diagnosing the present episode minimum five symptoms, which should be present most of the time for at least two weeks: altered mood in a depressive or expansive sense, low interest and pleasure for everyday activities, changes in weight, changes in sleep, changes in routine activity with fatigue or anergy, feelings of loss of self-worth or even guilt, decreased attention span, suicidal thoughts [20].

\section{ㅁ Prefrontal cortex (PFC)}

PFC is located anterior to the premotor area and the primary motor area. PFC has a multimodal function, integrating sensory-motor information with motivationalaffective function in the major depressive episode, being affected exactly this connectivity. PFC has three major sections: the dorsolateral prefrontal cortex (DLPFC), the orbital and medial paralimbic cortex, and the anterior cingulate cortex (ACC).

The ventromedial prefrontal cortex (VMPFC) is connected by the cingulate gyrus and hippocampus to the DLPFC. VMPFC has the functions of generating normal emotions, especially those with social connotations and for the regulation of autonomous and neuroendocrine responses, for the modulation of pain, for the expression of aggression, as well as for sexual and eating behaviors. Orbital PFC corrects the emotional or behavioral responses generated partially by the amygdala. DLPFC is involved in cognitive control, in solving complex tasks, in maintaining information in the buffer and manipulating it in working memory [21]. PFCs, the amygdala and especially the hippocampus are the brain structures considered to be most directly related to depressive pathology. Magnetic resonance imaging (MRI) studies support reduced brain volume in patients with depression compared to groups of healthy subjects, large decreases of the ACC, orbitofrontal cortex (OFC), and temperate decreases in the hippocampus, putamen, and caudate nucleus [21]. PFC has high levels of GRs and regulates HPA activity under stressful conditions and behaviors. All of the above support the association between dysfunctional pathology of PFC and MDD. Another significant argument in favor of PFC dysfunction in patients with MDD is the presence of ruminations along with impaired prosexual function and irrationally negative cognition in the perception of the future within MDD [18].

There are primary neurological diseases that cause secondary depression. In such clinical situations, the anatomical substrate is represented by the basal ganglia and the frontal cortex, especially the lesions that affect the striatal area or the left PFC lead to major depressions of organic cause. In clinical situations where major depression is primary, computed tomography (CT) examinations showed nonspecific differences, such as ventricular enlargement, global cortical atrophy, without explicitly highlighting any abnormalities in those regions. MRI studies of focal hyperactivity showed a more prevalent white matter lesions in territories linked to the frontal cortex and basal ganglia. Also, specific volumetric abnormalities involving the frontal cortex are noted in primary depression [22]. MDD implies a decrease in the ability to react to the reward and the left prefrontal hypoactivity observed in depression supports the behavioral deficit in approaching the rewardpunishment system. In a study on the effects of high frequency repetitive transcranial magnetic stimulation (HF-rTMS) treatment in treatment-resistant melancholic depression, it demonstrates the success of the repetitive transcranial magnetic stimulation approach as related to metabolic changes of the anterior cingulate gyrus (ACG) - Brodmann areas 24 and 32 [23].

\section{a Glial cells}

Post-mortem studies performed on patients with MDD have shown both a low number of glial cells and an alteration of their morphology, which is mainly found in PFC compared to other regions of the brain. Exposure to stress according to recent studies, induces pathologies in glial cells, a fact demonstrated by decreasing their density in the hippocampus and decreasing the density of astro- 
cytes in PFC in studies performed on animals exposed to chronic stress. The results suggest that glial function is deficient or even compromised in PFC and represents the anatomical substrate of depressive symptoms. We are talking here about a junctional intercellular communication that involves a gap between sections, a noticeable gap in astrocytes and which in turn leads to changes in neural function in PFC [18].

\section{a The limbic system}

In depression, we find hyperactivity in limbic areas, known to be associated with emotion processing. They are inhibited by the prefrontal areas in case they become inadequate. The above, support the limbic-cortical model encountered in depression, a well-established circuit that includes lateral PFC, medial PFC, OFC, ACC, hippocampus, thalamus, and amygdala. The cortico-striatal model highlights that the subcortical structures are important in information processing. There are overlapping cortical-striatalpallado-thalamic loops located in parallel, and any striatal dysfunction causes symptoms of psychomotor retardation. Reaction time is dependent on the level of oxygenation of the blood, which in turn regulates the concentration, stability, and selectivity of attention (blood oxygenation level-dependent-BOLD). An increased activity is found in the areas exposed above when the subject performs actions directed towards a goal, which involves both emotionality and cognition at the decision-making level. We find an impairment of BOLD, as well as a hypoactivity in the cortical areas described in clinically depressed people, compared to the control groups [20].

PFC has a significant inhibitory regulatory effect on limbic structures. When PFC is compromised in depression, the balance between the structures of the neurocircuit is disturbed due to decreased activity in the PFC. This dysfunction produces clinical symptoms in terms of behavior and intellect, neurophysiopathology being based on neuroendocrine disorders, neurotransmitter disorders, disorders of the autonomic system and immune dysfunctions, all of which are characteristic of MDD. Antidepressants increase the concentrations of monoaminergic neurotransmitters and can reverse structural changes leading to beneficial modulations on the disrupted PFC limbic neurocircuit [8].

\section{The amygdala}

The amygdala is involved in signaling recruitment and coordinating the cortical excitation response, which in turn produces a neuroendocrine response to nonspecific stimuli, such as surprising or ambiguous ones. The amygdala is involved in memory processes and learning through emotions. There has been found correlations between the hyperactivity in the amygdala and the intensity of depression, with the inclination towards ruminations and intrusive thoughts, with satellite anxiety. The above described are valid both in MDD from recurrent depressive disorder and in MDD from bipolar affective disorder [21]. Another study on 352 healthy participants based on structural neuroimaging found that early exposure to psychotraumatic events is associated with a lower volume of the amygdala in adolescence, a volume that decreases even more over time, as that group of subjects reaches adulthood [24]. Another meta-analysis performed on four studies that investigated amygdala volume in patients with posttraumatic stress disorder (PTSD) in adult life, but who were exposed to different types of abuse since childhood, reveals similar findings, namely that the group subjects with PTSD showed a significant bilateral reduction in amygdala volume compared to the control group of healthy subjects [14].

The amygdala plays a major role in expressing emotional expression, mood in situations of suffering or threat, the psychological part being involved in the previous organization of the endocrine response. There is a hyperactivity of the amygdala when the depressed patient is exposed to negative stimuli but also a hypoactivity at this level when the person in question benefits from positive stimuli. Stress hormones are secreted in excess in patients with MDD.

\section{Hippocampus}

One of the main studied structure is the hippocampus because of its correlation with depression, both in animal studies and in human studies. The reasons behind this are: the fundamental role of the hippocampus in learning and memory processes, hippocampal dysfunction is responsible for environmental and situation-dependent emotional responses, the hippocampus has many corticosteroid receptors, being related with the hypothalamus from anatomical and physiological point of view by a bunch of axons that penetrates through the fornix and reaches the HPA axis, providing inhibitory regulatory feedback. The hippocampus is one of the two areas of the brain where it is known that neurogenesis still continues in the mature brain in both animals and humans, dictating the high capacity of neuroplasticity of the hippocampus and the need for brain antidepressant medication with action at this level [21].

The opposition reaction from childhood, constantly present and at a formidable intensity, damages in time the microstructure of the hippocampus, affecting neurogenesis, as well as the survival of neurons and glial cells. Consequently, large-scale synaptic loss will occur, leading to damage to the cortisol-inhibited reaction loop in the HPA axis. At the same time, control over the secretion of corticosteroids in stress is disrupted. Thus, hyper-cortisol is due to the child's prolonged opposition disorder and results in negative feedback in the hippocampus. In turn, the hippocampus will exert a neurotoxic effect, which will lead to altered neuronal plasticity or directly to cellular apoptosis. MRI studies support significant changes in hippocampal volume in adults who were abused and abused in childhood, their hippocampal volume being significantly reduced compared to groups of subjects who were not exposed to stressors [14].

Impaired hippocampal function is reflected in impaired verbal memory test results in patients no matter the number of depressive episodes. The difference is, however, that hippocampal volume is reduced only in the group of patients with multiple depressive episodes, suggesting that hippocampal dysfunction precedes their detectable structural changes. In the case of patients treated with effective and potent antidepressants, in which we notice 
the clinical improvement of the symptomatology, we can later see the reversibility of the structural changes. In other words, patients with remitted depressive episodes have higher hippocampal volumes compared to patients with chronic depression. However, subjects who recovered without medication and emerged from a major depressive episode, known with a history of recurrent depressive history, are those with persistent small hippocampal volumes. It is thus suggested that the structural abnormallities of the hippocampus are a characteristic feature of depression. Another argument in the same direction is that in adolescents at high risk of depression, which is associated with psychotrauma in early childhood, hippocampal volumes are small, even if at the time of MRI measurements, the subjects do not show clinically manifest mood changes [25].

Neuroplasticity of antidepressants in the hippocampus works by linking: the hippocampus as a brain region extremely sensitive to stress-personality structure vulnerable to psychotrauma-MDD. The small volumes of gray matter in the hippocampus are primarily correlated with poor memory performance. There is a clinical debate about what changes occurred per primam: mood disorders versus disorders of congruent memory or explicit memory (decreasing reliability in solving the task). A significant role belongs to the medial temporal lobe, lesions that affect the expression of emotional behavior affecting the membranous-cortical-striatal-pallidal-thalamic circuits, circuits that include: PFC, amygdala, hippocampus, striated nucleus, ventromedial, mid-dorsal thalamic nucleus, midline, and the ventral pallidum nucleus.

Between $4-6 \%$ of subjects with MDD had smaller hippocampal volumes, the analysis included 1167 depressed patients and 1088 control subjects with a wide age range from pediatrics to the geriatric population [23].

\section{ㅁ Cortical gray matter}

Childhood opposition disorder has long-term detrimental effects on many higher gnostic functions, such as working memory and learning, but also on other processes: inhibitory control, prosexual function, emotion processing, reward motivation system. Dysfunctions at this level not only lead to impaired intelligence quotient (IQ) or academic achievement, but also determine issues related to emotion regulation or impulse control. In other words, structural neuroimaging studies have shown that the opposition disorder present in childhood correlates in adult life with gray matter abnormalities in various cortical regions, with changes in volume and impairment of neural circuits of emotional processing, motivation-reward or knowledge (higher cognitive functions of planning, sequencing, operationalization, control, and feedback). The neuroanatomical structures involved are ACC, DLPFC, OFC, VMPFC [14].

When we refer to the other range of age (geriatric patients) with late-onset depression, it has been shown gray matter loss up to $20 \%$ in the right parietal cortex and going up to $10 \%$ decrease in posterior portions of the superior and inferior temporal gyrus. The researchers used a threedimensional (3D) vector deformation field technique based on MRI scans [26].

\section{ㅁ Subcortical abnormalities of the white matter}

Until recently, studies focused mainly on gray matter in the depression neurocircuit, neglecting connections to the subcortical white matter. Abnormalities at this subcortical level have been described in depressed elderly people (lateonset depression), which initially led to the idea that it is a neurological problem associated with ischemic vascular pathology. MRI signal show that in the prefrontal area there are many hyperdensities of white matter, being associated with low metabolism in the frontal lobe and cortical atrophy [21]. Opposition disorder present in childhood is considered to be associated with low structural connectivity of several white matter neural tracts located at the following levels: corpus callosum, hooked bundle, cingulum, fornix, upper longitudinal bundle, lower longitudinal bundle, inferior fronto-occipital bundle. We find the anomalies described above in both children and adolescents and adults. Of all, the decrease in the integrity of the corpus callosum is the most consistent finding. The corpus callosum is a broad commissure fiber, which connects the cortical cortex of the two hemispheres, having a crucial role in ensuring the integrity of interhemispheric communication in terms of processing higher cognitive functions but also processing perception and arousal, as well as everything that means emotional-motivational-volitional regulation. The corpus callosum integrity decreases in people who experienced opposition disorder or early depression [14].

The progression of white matter changes has been associated with cardiovascular risk factors especially with high blood pressure and smoking, and with age. The severity of the changes is associated with a poor response to treatment and the development of new depressive symptoms despite the timely introduction of appropriate, long-term treatment, the mood deteriorating. Despite these findings, the direct causality between white matter changes and depression remains unclear, as most studies are conducted in cross section. However, changes in white matter have a negative influence on the progression of depression. There remains the possibility that both white matter changes and depressive symptoms may be based on a common pathological origin, and depressive symptoms may precede the development of white matter changes in a manner similar to how cardiovascular disease precedes stroke [27].

\section{ㅁ Immunological mechanisms}

The complex pathophysiology of depression is linked with immunological mechanisms from the idea that proinflammatory cytokines (signaling molecules of the immune system) cause fatigue and fatigue followed by anxiety and depression, which can lead to clinically obvious depression. It is known that patients receiving interferon (IFN) treatments for other systemic conditions are at risk of decompensating with depression. The immune activation and especially increased cytokines concentrations is associated with severe depression [21].

Rodent studies have shown that cytokines can induce depressive behavior, suggesting causal links between inflammation and depressive mood swings. Cytokines, including interleukin (IL) 1, IL6, IFN, and tumor necrosis factor alpha $(\mathrm{TNF} \alpha)$, have been shown in preclinical and 
clinical studies to be involved in the development of a depressive pattern dominated by anhedonia, fatigue, bradykinesia, decreased appetite, and sleep disturbances. If anti-inflammatory cytokines, cytokine receptor antagonists or IL1 receptor antagonists (IL1-RA) are administered in those clinical situations, the behavior of depressive disorder may be ameliorated. A recent meta-analysis performed on dispositional normal subjects revealed that a significant increase in TNF $\alpha$, IL6 and C-reactive protein (CRP) may indicate depression [28]. Another review suggests a significant association between interferon-gamma (IFN- $\gamma$ ) and IL2 [13].

The HPA axis releases corticotropin-releasing hormone (CRH) from the hypothalamus in response to stress. $\mathrm{CRH}$ stimulates the secretion of adrenocorticotropic hormone (ACTH) from the pituitary gland, which in turn releases glucocorticoids from the adrenal glands. Glucocorticoids act through two receptors: mineralocorticoid receptor (MR), with high affinity for glucocorticoids and GR, with low affinity for glucocorticoids. An increase in glucocorticoid levels saturates MRs and activates GRs, which causes negative feedback on the HPA axis. Both GR and MR are present in high concentrations in PCF and hippocampus. That is why it has been suggested that they are strongly involved in the pathology of depression. On the other hand, for over 50 years, it has been known that a prolonged increase in cortisol causes neuronal dysfunction by decreasing glucose absorption, reducing dendritic arborization, apoptosis or cell loss in PFC and hippocampus [18].

The autonomic nervous system and the HPA axis are directly related to the concept of stress. On the other hand, in a secular view, depression is considered to be caused by a psychotraumatic factor, in other words "everything starts with an upset". Healing a depressive episode involves not only alleviating the clinical symptoms of depression but also eradicating the alleged pathogen from the subject's mind (development of psychotrauma). It remains a discussion in itself the breakdown of the concept of stress between the distress that produces the mentioned functional and neuroanatomical changes and the stress that is necessary for the optimal state of preparation of the organism for the success in the current activities.

\section{a Neurochemistry and neurotransmitters}

Classic tricyclic antidepressants act on a multitude of receptors, with significant correlations not only with serotonergic and noradrenergic receptors, but also with histamine and muscarinic ones. The importance of the enteric system and the disposition of the receptors at that level should not be neglected, the correspondent being the multitude of gastrointestinal symptoms in major depression. We should also discuss the current trend of young adults in the US to abuse antidepressants (in a manner similar to antibiotic abuse), in an empirical desire to "tune" their body not only for adaptive social-professional functionality, high but also for strengthening immune mechanisms.

Understanding the mechanism of action of antidepressants must start from understanding the pathogenesis of depression. The early hypothesis that survives to this day is that of "monoamine depression", in other words depression as a disorder is caused by a deficiency of a monoamine, be it serotonin (5-hydroxytriptamine - 5-HT) (mild to moderate intensity anxiety) or noradrenergic type (apatabolic staining, endogenous expression).

Subsequent animal studies show changes in density at other adrenergic and serotonergic receptors, as well as changes in receptor sensitivity in areas located in the terminal between the neuronal and the soma, and postsynaptic. Adaptive mechanism that responds when the neurotransmitter availability increases is associated with receptor changes [29].

Research on people who are healthy in terms of mood (euthymic) have shown that the administration of antidepressants has acted on the adrenergic system by increasing its activity. Thus, the beta receptor regulation hypothesis was not supported.

Studies made on animals have shown that, despite a reduction in post-synaptic beta-adrenoreceptor density, increases in associated intracellular activity (protein synthesis) and hypoactivation of the post-receptor signal transduction cascade have been found. These findings refine the monoamine hypothesis by demonstrating that antidepressants work by increasing the synaptic transmission of the chemical neurotransmitter. There are studies highlighting the changes in serotonin concentrations and norepinephrine (noradrenaline -NA) metabolites, decreases of their values, showing that neuroendocrine responses to serotonergic and noradrenergic receptor stimulation have been unsuccessful, with depressive symptoms recurring. This is the case of treatmentresistant depression or depression in which relapses occur during the same episodes when in the first weeks of treatment the results are effective for the antidepressants used and yet, relapse cannot be prevented. There are also clinical situations in which depressed patients are treated with Tryptophan (5-Hydroxytryptophan - 5-HTP) or alphamethyl-para-tyrosine (AMPT), which reduce the concentration of serotonin and NA and support the hypothesis that the transmission of these neurotransmitters is affected in depression [21].

Because the neurobiochemical serotonin deficit in the pathogenesis of the major depression is overemphasized and the selective serotonin reuptake inhibitors and related antidepressants are extensively administered in clinical practice, the negative consequence is a decreasing of the dopamine (DA) transmission. This phenomenon is based on the decreased firing in the dopaminergic neurons from the hippocampal system and from the basolateral amygdala.

This reduced dopaminergic neurotransmission efficacy may be augmented by the association of antipsychotics. Lower dopaminergic system efficacy may be an important factor for the onset of pharmacological resistance in MDD, due to dysconnectivity between hippocampal and amygdala with the thalamus and frontal cortex. As clinical consequences of this phenomenon, persistent anhedonia syndrome and treatment non-responsivity have been reported [30]. This disconnective pattern can be frequently associated in the elderly with the onset of cognitive decline and rapid progression of invasion of neurodegenerative elements into the cortico-hippocampal cognitive circuits in Alzheimer's disease [31].

In humans, the solute carrier family 6 member 4 (SLC6A4) gene is the transporter of serotonin and is located on chromosome 17q11.1-17q12. It encodes 5-hydroxytryptamine (serotonin) transporter (5-HTT), which is involved 
in absorbing serotonin in serotonergic neurons. Deleting or inserting a single other element in tandem with 44 bases and this happens in the region of the 5'-flanked promoter of this gene, 5-hydroxytryptophan-transporter-linked polymorphic region $(5-H T T L P R)$, produces a short $(S)$ or long $(L)$ allele. The 5-HTTLPR $S$ allele is linked with reduced tryptophan expression and consequently decreased serotonin uptake activity. It has been demonstrated that one or two copies of the $S$ allele are associated with a higher risk of developing depression if the genetic component interacts with inappropriate psychosocial environments, such as stressful, early, or late life events, or opposition disorder from an early age [14].

Brain-derived neurotrophic factor (BDNF) appears to be a crucial link in the neurobiology of depression, referring to effects of antidepressant drugs, neurotransmitter function, HPA abnormalities [21].

The hippocampus, that plays a major role in neuronal growth, is rich in BDNF. It also plays an important role in neuronal survival and maturation, dendrite arborization, and synaptic neuroplasticity in the brain of adults. Stress suppresses BDNF synthesis in the hippocampus, while antidepressants increase synthesis by sending signals to PFC and hippocampus. In patients with depression, serum BDNF levels are low in proportion to the severity of the depression. These concentrations increase as antidepressants are administered or electroconvulsive therapy (ECT) sessions are performed. Post-mortem studies in depressed patients at death revealed significant differences in hippocampal BDNF concentrations between treated patients and those who did not accept antidepressant medication.

\section{口 Genetic aspects}

Two of major risk factors in MDD are represented by genetic factors and a positive family history of depression. There are also intermediate concepts, such as that of heritability or that of family agglomeration or that of cross-inheritance between various psychiatric disorders (e.g., between MDD and alcoholism). Having parents diagnosed with depression increases three times the risk of offspring in terms of developing a depressive episode. Research claims that family history is not exclusively related to symptoms but even associated with neuroanatomical structural changes [15].

In depression, the influence of environmental factors is strong related to it and the current model according to which specialists judge each clinical case is the diathesisstress, which examines the interaction of genes with the environment. Therefore, for depressed patients, exposed to early pshychotrauma, serotonin receptor $3 \mathrm{~A}$ genotype (HTR3A-42C.T) has been linked to a loss of gray matter in the hippocampus and PFC [21]. The interaction of environmental factors is also supported by the fact that in approximately $7-8 \%$ of cases, the first episode of MDD is preceded by a severe stressful life event [18], after which the following depressive episodes can be determined by intensity stressors from smaller and smaller (endoreactivity hypothesis). The regulatory model of the system on the HPA axis in depression includes atypical responses to Dexamethasone, increased basal cortisol levels and hyperreactivity to stressors at psychological level. This leads to abnormalities in the negative feedback system of the
HPA axis, as well as abnormalities in the production of hormones in the range of corticotrophins (CRH). We also notice a sensitivity of the pituitary gland in the conditions of an intact adrenal gland. In MDD patients (both periods of depression and remission), studying the peripheral blood cells, a reduced messenger ribonucleic acid (mRNA) expression of the GR- $\alpha$ - the isomorphic GR that binds glucocorticoids. In addition, grade 1 relatives of patients with bipolar affective disorder also show a reduction in mRNA and GR- $\alpha$, findings that, taken together, suggest abnormalities of the HPA axis at least at the GR level and can be considered an important marker in disorders of disposition.

Depressed patients with childhood abuse history, if subjected to a new psychosocial stressor in adulthood, when a low-dose Dexamethasone is administrated, improved their HPA axis responses as well as the ACTH and cortisol response. The same results are not found in patients with MDD who do not have a history of childhood abuse. High cortisol responses to Dexamethasone or $\mathrm{CRH}$ have been found to healthy subjects that have a high genetic risk for affective disorders have. There are significant differences between the extent of such responses in healthy subjects and without a family history of depression compared to patients who manifest overt clinical symptoms. The increased reactivity of patients with depression to Dexamethasone or $\mathrm{CRH}$ compared to the control group remained at the same high levels for a period of four years of follow-up. According to these studies, genetic vulnerability has a key role in depression rather than early life experience [21].

\section{ㅁ Psychopharmacological intervention in depression}

Plasticity helps the brain recover from damage caused by events such as strokes or head trauma. Plasticity implies the ability to manipulate specific neural pathways and synapses, to receive new functions and implications through psychotherapeutic or psychopharmacological interventions.

Studies on brain adaptation demonstrate the existence of four major forms of functional neuroplasticity studied in humans: ( $i$ ) taking over the functions by the homologous area; (ii) cross-reassignment; (iii) extension of cortical mapping corresponding to a specific sensory or motor homunculus-compensatory masquerade.

The adaptation of the homologous zone supposes the assumption of the respective cognitive process will be taking over by the opposite hemisphere through a homologous region. Cross-reassignment appears when structures previously devoted to the processing of a particular type of sensory input now accept the receipt of that input through a new sensory mode. The extension of the brain map involves the enlargement of a functional region of the brain from close to close, by accumulating new performances of areas that previously did not deal with that function or mental process. Compensatory masquerade involves the allocation of a certain cognitive process to a new area of the brain that previously did not perform those tasks.

When in adult life the neurons are generated from neuronal stem cells, we assist to a process called neurogenesis. This genesis differs from neurogenesis in intrauterine life. Many authors consider that stress is the most important factor for the onset of depression after genetic 
predisposition [32]. Hippocampal cells are sensitive to stress and the risk is decreased neurogenesis at exactly this level where the process itself is vital. Studies in rats whose adrenal glands have been removed have shown, in the dentate gyrus, an increased neurogenesis process. The response to a stressor that is highlighted by the cortisol production is linked to the adrenal gland. When cortisol is produced in large quantities and in a chronic manner, it causes down-regulation of serotonergic receptors and suppresses neurogenesis. In studies in normal animals, corticosterone has been shown to suppress neurogenesis.

The first step in the administration of antidepressants is selective serotonin reuptake inhibitors (SSRIs). The neurogenesis can explain the efficiency of SSRIs. In a normal brain, increased serotonin suppresses the hormone that releases $\mathrm{CRH}$ through action in the hippocampus. The action is done directly on the paraventricular nucleus, to reduce the release of $\mathrm{CRH}$ and downregulate the discharge of NA from the locus coeruleus. Neurogenesis involves the production of several brain cells, especially by acting on the 5-HT1A receptor in the dentate gyrus of the hippocampus, which improves the symptoms of depression. Normally, neurogenesis requires between 3-6 weeks for the maturation of newly formed neurons, this being exactly the time required for SSRIs to act. The correlation reinforces the hypothesis that SSRIs reduce the symptoms of depression through the mechanism of neurogenesis.

\section{a Modulation of the brain network by treatment}

New dentate granule cells (DGCs) capable of processing information are generated by the dentate gyrus of the adult hippocampus. In adult neurogenesis, neuronal progenitor cells take a new path, migrate, and mature over several weeks. At the same time, the young DGCs expand their axon and dendrites by establishing an adequate inputoutput connectivity within the pre-existing network. Thus, there are improvements in cognitive deficits in depression, even if the regions of the brain have suffered structural damage. ECT is perhaps the most effective antidepressant therapy, with the most profound effects on the mechanisms of brain neuroplasticity. Unfortunately, the short-term retrograde amnesia that occurs after each ECT session makes it difficult to examine cognitive improvement in depressed patients treated with ECT. There are also studies that support the improvement of memory and other cognitive functions in depressed patients after drug treatment. Although currently withdrawn from the Romanian pharmacological market, Moclobemide, as a standard drug for monoamine oxidase inhibitors (MAOIs), in studies conducted abroad, has been shown to be superior to Viloxazine (adrenergic agonist) or Maprotiline (tetracyclic antidepressant) [31]. In the present studies, superiority refers to improved memory. Studies have shown that for MDD patients, treated with Fluoxetine, the verbal memory improved. The patients with late-onset depression after treatment with Fluoxetine or Reboxetine have shown an improvement in memory and attention. The limitations of this study come from the absence of a control group that was treated with placebo [33].

Stress can cause atrophy of both the hippocampus and PFC, with morphological changes in both regions being observed in patients with depression. Under these conditions, an ideal antidepressant should prevent cortical atrophy and stimulate the mechanisms of neurogenesis. Some studies that examine the volume of the hippocampus in patients with a long history of depression, find correlations with the time spent by the patient in symptomatic conditions. This suggests that a successful treatment quickly reduces symptoms and stops the deteriorating processes. Even in patients with PTSD, where we also find a reduction in hippocampal volume, antidepressants improve declarative memory.

\section{a Conclusions}

Cerebral neuroplasticity is the central axis of the remission of MDD and the fundamental mechanism by which neurons defend themselves against stress. HPA function requires changes in GR density. Dysfunction delays synaptic transmission in the hippocampus. Childhood abuse, neglect, or opposition disorder are risk factors for depression in a busy life. Some epigenetic changes, such as DNA methylation, can persist into adulthood and dictate personal vulnerability to stress. This is correlated with the activity of the HPA axis. In depression there are hyperactivity in the amygdala, striated nucleus, limbic and subcortical regions. Not only antidepressants but also psychotherapy can alter the functionality of the brain. Neurons have their own ability to change their power in terms of the efficiency of synaptic transmission. At the neurobiological level, we are talking about the remodeling of interconnectivity and at the psychological level, we are talking about active will. PFC has a multimodal function being focused on the connectivity between the sensorymotor information with the motivational-affective ones. In major depression, there is a dysfunction of PFC proven by ruminations, negative cognition, and negative perception of the future, as well as disorders of concentration, stability, and selectivity of attention.

In major depression, there is a hyperactivity of the white matter in the territories related to the frontal cortex and basal ganglia. Major depression involves altering the reward-punishment system. HF-rTMS produces metabolic changes in the ACG. Post-mortem in major depression, a reduced number of glial cells was found in the PFC; here there is also a dysfunctional junctional intercellular communication, which consists in a visible decrease in the transmission of the signal between astrocytes. Depression involves hyperactivity in the limbic areas where emotions are processed, with inhibition of the prefrontal areas. Hyperactivity is found especially in the amygdala, correlating directly with ruminant tendencies, intrusiveness, and adjacent anxiety. The amygdala is a key point in modulating affectivity. The hippocampus, which has numerous receptors for corticosteroids, is responsible for reactive-mediated emotional responses to life situations. The hippocampus is also the area where neurogenesis continues in the mature brain, being the area with high neuroplasticity. Opposition disorder in childhood causes decreased hippocampus, impaired neurogenesis, impaired glial cells, synaptic impairment, all of which ultimately lead to HPA axis dysfunction. MRI studies support low hippocampal volume in adults known as former abused children. The reduction of the hippocampal volume occurs 
only in the case of multiple depressive episodes or in patients left untreated. When effective antidepressants are used, structural changes are reversible. Impaired hippocampal function correlates with poor results on verbal memory tests. Dysfunction of cortical gray matter correlates with IQ damage, emotion regulation, or impulse control. White matter abnormalities are found in the elderly, as well as in subjects at cardiovascular risk. These subjects have a poor response to medication and are at risk of developing new depressive symptoms. Subjects with childhood opposition disorder or depression that occurred at an early age affected the integrity of the corpus callosum. Proinflammatory cytokines (IL1, IL6, IFN and TNF $\alpha$ ) are involved in the development of a depressed pattern dominated by the apatho-abulic component and anti-inflammatory cytokines, cytokine receptor antagonists or IL1 receptor antagonists are administered for amelioration.

Serotonergic depression has the following stages of increase: SSRIs; serotonin antagonist and reuptake inhibitors (SARIs); 5HT1 receptor agonist (Buspirone has full agonist on 5HT1A somato-dendritic receptors and partial agonist on 5HT1A receptors; the mechanism is unknown, maybe linked to release of DA and NA in PFC [24]; although Buspirone also has D2 receptor antagonism, it is not considered or used as a neuroleptic because it has no clinical antipsychotic effects); association of thyroid hormones; 5HT1A receptor antagonist (Pindolol -5 HT1A receptor and $\beta$-adrenergic antagonist mainly on serotonin presynaptic autoreceptors thus blocking the self-inhibition of serotonergic transmission; it has no action on postsynaptic serotonin receptors; 5-HT2A serotonin receptor (Fluphenazine is a 5-HT receptor agonist/antagonist, used in the therapy of obesity, in the therapy of childhood autism and in bipolar disorders, as a predictor of Lithium prophylaxis for the manic phase; it acts through its active metabolism, with a half-life of 11-20 hours).

The noradrenergic depression, in which the steps of increase would be: inhibitors of noradrenaline (NA) reuptake (Reboxetine, Desipramine, Maprotiline); inhibitors of NA and DA reuptake (Bupropion); associations with previous medication by: dopaminergic agonists (Bromocriptine), association of thyroid hormones, presynaptic DA release [Pemoline is a noradrenergic-type psychostimulant agent used in post-ECT mental disorders and adjuvant in the therapeutic management of depressive states, hyperkinetic syndromes, attention deficit hyperactivity disorder (ADHD) and narcolepsy]; Amphetamine or Methylphenidate supplementation.

The adaptive deficit may be correlated with the triggering of apoptotic mechanisms and the installation of irreversible cerebral structural alterations. Thus, the use of antidepressants without anticholinergic effect, as well as the new classes of medication, ensures the prophylaxis of structural changes at the cortical level.

BDNF is a major link in the neurobiology of depression. Structural neuroplasticity is involved in the neurogenesis of adult subjects through mechanisms such as dendritic reconstruction, and functional neuroplasticity involves molecular and cellular mechanisms at the synaptic level.

\section{Conflict of interests}

The authors declare that they have no conflict of interests.

\section{References}

[1] Patten SB, Kennedy SH, Lam RW, O'Donovan C, Filteau MJ, Parikh SV, Ravindran AV; Canadian Network for Mood and Anxiety Treatments (CANMAT). Canadian Network for Mood and Anxiety Treatments (CANMAT) clinical guidelines for the management of major depressive disorder in adults. I. Classification, burden and principles of management. J Affect Disord, 2009, 117(Suppl 1):S5-S14. https://doi.org/10.1016/j.jad.2009. 06.044 PMID: 19674796

[2] Gilmour H, Patten SB. Depression and work impairment. Health Rep, 2007, 18(1):9-22. PMID: 17441440

[3] Murrough JW, lacoviello B, Neumeister A, Charney DS, losifescu DV. Cognitive dysfunction in depression: neurocircuitry and new therapeutic strategies. Neurobiol Learn Mem, 2011, 96(4):553-563. https://doi.org/10.1016/j.nlm.2011.06. 006 PMID: 21704176

[4] Hasselbalch BJ, Knorr U, Hasselbalch SG, Gade A, Kessing LV. Cognitive deficits in the remitted state of unipolar depressive disorder. Neuropsychology, 2012, 26(5):642-651. https://doi.org/ 10.1037/a0029301 PMID: 22823136

[5] Hawton K, Casañas I, Comabella C, Haw C, Saunders K. Risk factors for suicide in individuals with depression: a systematic review. J Affect Disord, 2013, 147(1-3):17-28. https://doi.org/10.1016/j.jad.2013.01.004 PMID: 23411024

[6] Gournellis R, Tournikioti K, Touloumi G, Thomadakis C, Michalopoulou PG, Michopoulos I, Christodoulou C, Papadopoulou A, Douzenis A. Psychotic (delusional) depression and completed suicide: a systematic review and meta-analysis. Ann Gen Psychiatry, 2018, 17:39. https://doi.org/10.1186/ s12991-018-0207-1 PMID: 30258483 PMCID: PMC6150953

[7] Gold PW, Machado-Vieira R, Pavlatou MG. Clinical and biochemical manifestations of depression: relation to the neurobiology of stress. Neural Plast, 2015, 2015:581976. https://doi.org/10.1155/2015/581976 PMID: 25878903 PMCID: PMC4387963

[8] Łojko D, Rybakowski JK. Atypical depression: current perspectives. Neuropsychiatr Dis Treat, 2017, 13:2447-2456. https:// doi.org/10.2147/NDT.S147317 PMID: 29033570 PMCID: PMC5614762

[9] Patel RK, Rose GM. Persistent depressive disorder (dysthymia). 2020 Jun 29. In: ${ }^{* * *}$. StatPearls [Internet]. StatPearls Publishing, Treasure Island, FL, USA, 2020 Jan-. PMID: 31082096 NBK541052

[10] Byers AL, Covinsky KE, Barnes DE, Yaffe K. Dysthymia and depression increase risk of dementia and mortality among older veterans. Am J Geriatr Psychiatry, 2012, 20(8):664-672. https:// doi.org/10.1097/JGP.0b013e31822001c1 PMID: 21597358 PMCID: PMC3229643

[11] Hantsoo L, Epperson CN. Premenstrual dysphoric disorder: epidemiology and treatment. Curr Psychiatry Rep, 2015, 17(11):87. https://doi.org/10.1007/s11920-015-0628-3 PMID: 26377947 PMCID: PMC4890701

[12] American Psychiatric Association (APA). Diagnostic and statistical manual of mental disorders (DSM-5). $5^{\text {th }}$ edition, APA, Arlington, VA, USA, 2013. https://doi.org/10.1176/appi. books.9780890425596

[13] Eyre H, Baune BT. Neuroplastic changes in depression: a role for the immune system. Psychoneuroendocrinology, 2012, 37(9):1397-1416. https://doi.org/10.1016/j.psyneuen.2012.03. 019 PMID: 22525700

[14] Kim YK, Ham BJ, Han KM. Interactive effects of genetic polymorphisms and childhood adversity on brain morphologic changes in depression. Prog Neuropsychopharmacol Biol Psychiatry, 2019, 91:4-13. https://doi.org/10.1016/j.pnpbp. 2018.03.009 PMID: 29535036

[15] Carballedo A, Lisiecka D, Fagan A, Saleh K, Ferguson Y, Connolly G, Meaney J, Frodl T. Early life adversity is associated with brain changes in subjects at family risk for depression. World J Biol Psychiatry, 2012, 13(8):569-578. https://doi.org/10.3109/15622975.2012.661079 PMID: 22515408

[16] Wiswede D, Taubner S, Buchheim A, Münte TF, Stasch M, Cierpka M, Kächele H, Roth G, Erhard P, Kessler H. Tracking functional brain changes in patients with depression under psychodynamic psychotherapy using individualized stimuli. PLoS One, 2014, 9(9):e109037. https://doi.org/10.1371/journal. pone.0109037 PMID: 25275317 PMCID: PMC4183537 
[17] Jorgensen A, Magnusson P, Hanson LG, Kirkegaard T, Benveniste $H$, Lee $H$, Svarer C, Mikkelsen JD, Fink-Jensen A, Knudsen GM, Paulson OB, Bolwig TG, Jorgensen MB. Regional brain volumes, diffusivity, and metabolite changes after electroconvulsive therapy for severe depression. Acta Psychiatr Scand, 2016, 133(2):154-164. https://doi.org/10. 1111/acps.12462 PMID: 26138003

[18] Kupfer DJ, Frank E, Phillips ML. Major depressive disorder: new clinical, neurobiological, and treatment perspectives. Lancet, 2012, 379(9820):1045-1055. https://doi.org/10.1016/S01406736(11)60602-8 PMID: 22189047 PMCID: PMC3397431

[19] Sibille E. Molecular aging of the brain, neuroplasticity, and vulnerability to depression and other brain-related disorders. Dialogues Clin Neurosci, 2013, 15(1):53-65. PMID: 23576889 PMCID: PMC3622469

[20] Palmer SM, Crewther SG, Carey LM; START Project Team. A meta-analysis of changes in brain activity in clinical depression. Front Hum Neurosci, 2015, 8:1045. https://doi.org/10.3389/ fnhum.2014.01045 PMID: 25642179 PMCID: PMC4294131

[21] Palazidou E. The neurobiology of depression. Br Med Bull, 2012, 101(1):127-145. https://doi.org/10.1093/bmb/lds004 PMID: 22334281

[22] Meisterernst J, Klinger-Gratz PP, Leidolt L, Lang MF, Schroth G, Mordasini P, Heldner MR, Mono ML, Kurmann R, Buehlmann M, Fischer U, Arnold M, Gralla J, Mattle HP, El-Koussy M, Jung S. Focal T2 and FLAIR hyperintensities within the infarcted area: a suitable marker for patient selection for treatment? PLoS One, 2017, 12(9):e0185158. https://doi.org/10.1371/journal. pone.0185158 PMID: 28957339 PMCID: PMC5619762

[23] Baeken C, De Raedt R, Van Hove C, Clerinx P, De Mey J, Bossuyt A. HF-rTMS treatment in medication-resistant melancholic depression: results from ${ }^{18} \mathrm{FDG}-\mathrm{PET}$ brain imaging. CNS Spectr, 2009, 14(8):439-448. https://doi.org/10.1017/ s1092852900020411 PMID: 19890238

[24] Korgaonkar MS, Antees C, Williams LM, Gatt JM, Bryant RA, Cohen R, Paul R, O'Hara R, Grieve SM. Early exposure to traumatic stressors impairs emotional brain circuitry. PLoS One, 2013, 8(9):e75524. https://doi.org/10.1371/journal.pone. 0075524 PMID: 24073270 PMCID: PMC3779182

[25] MacQueen G, Frodl T. The hippocampus in major depression: evidence for the convergence of the bench and bedside in psychiatric research? Mol Psychiatry, 2011, 16(3):252-264. https://doi.org/10.1038/mp.2010.80 PMID: 20661246
[26] Ballmaier M, Kumar A, Thompson PM, Narr KL, Lavretsky H, Estanol L, Deluca H, Toga AW. Localizing gray matter deficits in late-onset depression using computational cortical pattern matching methods. Am J Psychiatry, 2004, 161(11):20912099. https://doi.org/10.1176/appi.ajp.161.11.2091 PMID: 15514411

[27] Firbank MJ, Teodorczuk A, van der Flier WM, Gouw AA, Wallin A, Erkinjuntti T, Inzitari D, Wahlund LO, Pantoni L, Poggesi A, Pracucci G, Langhorne P, O’Brien JT; LADIS group. Relationship between progression of brain white matter changes and late-life depression: 3-year results from the LADIS study. Br J Psychiatry, 2012, 201(1):40-45. https://doi.org/10.1192/ bjp.bp.111.098897 PMID: 22626634

[28] Ng A, Tam WW, Zhang MW, Ho CS, Husain SF, Mclntyre RS, Ho RC. IL-1 $\beta$, IL-6, TNF- $\alpha$ and CRP in elderly patients with depression or Alzheimer's disease: systematic review and meta-analysis. Sci Rep, 2018, 8(1):12050. https://doi.org/ 10.1038/s41598-018-30487-6 PMID: 30104698 PMCID: PMC6089986

[29] Chai GS, Wang YY, Yasheng A, Zhao P. Beta 2-adrenergic receptor activation enhances neurogenesis in Alzheimer's disease mice. Neural Regen Res, 2016, 11(10):1617-1624. https://doi.org/10.4103/1673-5374.193241 PMID: 27904493 PMCID: PMC5116841

[30] Marinescu I, Vasiliu O, Vasile D. Translational approaches in treatment-resistant depression based on animal model. Rom J Morphol Embryol, 2018, 59(3):955-964. PMID: 30534840

[31] Marinescu I, Enătescu VR, Ghelase ŞM, Marinescu D. Neurobiological arguments for a pathogenic multifactorial disconnective model of cognitive disorders from Alzheimer's disease in elderly people. Rom J Morphol Embryol, 2017, 58(4):1165-1173. PMID: 29556605

[32] Pittenger C, Duman RS. Stress, depression, and neuroplasticity: a convergence of mechanisms. Neuropsychopharmacology, 2008, 33(1):88-109. https://doi.org/10.1038/sj.npp.1301574 PMID: 17851537

[33] Krysta K, Krzystanek M, Janas-Kozik M, Klasik A, KrupkaMatuszczyk I. Impact of pharmacological and psychological treatment methods of depressive and anxiety disorders on cognitive functioning. J Neural Transm (Vienna), 2015, 122(Suppl 1):S101-S110. https://doi.org/10.1007/s00702-0141282-3 PMID: 25078256 PMCID: PMC4529445

\section{Corresponding author}

Arina Cipriana Trifu, MD, Department of General Medicine, Medical Military Institute, 3-5 Medical Military Institute Street, Sector 1, 010919 Bucharest, Romania; Phone +40727-803 330, e-mail: arinatrifu@gmail.com 